\title{
RELATION BETWEEN PHYSICAL ACTIVITIES AND BUILT ENVIRONMENT: A LITERATURE REVIEW
}

\author{
Md. Masud Parves Rana \\ Associate Professor, Department of Geography and Environmental Studies \\ Rajshahi University, Rajshahi-6205, Bangladesh.
}

and

\section{Xiaolu Zhou}

PhD student, Department of Landscape Architecture, 101 Temple Hoyne Buell Hall 611 Lorado Taft Drive, MC-620, Champaign, Illinois 61820, USA

\begin{abstract}
Physical inactivity has been a leading factor of chronic diseases and high rate of mortality in the world. Despite the fact, only a small portion of people are able to meet up the recommended physical activities. However, there are handful studies suggest that built environment may provide stimulus or barriers to people's participation in physical activities. Drawing upon this context, this paper aims to review articles regarding 'built environment and physical activity' focusing on characteristics of built environment which are particularly helpful in improving neighborhood environment, and to catch the attention to physical activities, such as walking, and cycling. It also critically reviews the measures of built environment, and finds three measures viz. (i) perceived environment measures, (ii) observational measures, and (iii) GIS-based measures. The article argues that integrated measures of built environment might be helpful to reduce limitations of individual measures and to understand the reasons of less participation in physical activities. It also suggests some practical interventions for improvement of built environment which is essentially inevitable to persuade physical activities.
\end{abstract}

Keywords: Physical activities, built environment, measures, and practical interventions.

সারাংশ: বিশ্বে মরণব্যাধি ও অধিক হারে মরণশীলতার জন্য শারীরিক অকর্ম একটি প্রধান নিয়ামক। এ সত্ত্তেও, খুব অল্প সংখ্যক মানুষ সুপারিশকৃত শারীরিক কর্মকাঞ্ত (ব্যায়ানে) অংশগ্রহণ করতে পারে। যাই রোক, অনেক গবেষণা সুপারিশ করে যে, স্থাপিত পরিবেশ মানুযের শারীরিক ব্যায়ানে অংশগ্রহণে উদ্দীপক অথবা বাঁধা প্রদান করতে পারে। এই প্রেক্ষিতে, বর্তমান প্রবন্ধটি ‘স্থাপিত পরিবেশ এবং শারীরিক ব্যায়াম’ সম্পর্কিত গবেষণাসমূহ পর্যালোচনা করে যা মূলতঃ স্থাপিত পরিবেশের বৈশিষ্ট্যসমূহ, যেগুলো প্রতিবেশ উন্নয়নে সাহায্য করে; এবং শারীরিক ব্যায়াম (যেমন, পায়ে হাঁটা, দ্বিচক্রযান চালনা ইত্যাদি) এর প্রতি মনোযোগী করে তোলে। প্রবন্ধটি স্থাপিত পরিবেশ সম্পর্কিত সাধারণ পরিমাপকসমূহ; যেমন (ক) প্রত্যক্ষণকৃত পরিবেশ পরিমাপক, (খ) পর্যবেক্ষণমূলক পরিমাপক, এবং (গ) জিআইএস-নির্ভর পরিমাপক) পর্যালোচনা করে। তাছাড়াও এটি একটি সমন্বিত স্থাপিত পরিবেশ পরিমাপক কাঠানো উপস্থাপন করে যা স্বতন্ত্র পরিমাপকসমূহের দূর্বলতা হ্রাস করতে পারে এবং শারীরিক ব্যায়ান্ কম অংশগ্রহণের কারণসমূহ বুঝতে সাহায্য করে। এছাড়াও প্রবন্ধটি স্থাপিত পরিবেশ উন্নয়নে আরও কয়েকটি ব্যবহারিক সুপারিশ প্রদান করে, যেগুনো শারীরিক ব্যায়ানে অংশগ্রহণ বাড়াতত সহায়ক।

\section{Introduction}

Physical inactivity, being the second leading modifiable risk for chronic disease, accounts for a significant amount of mortality in the Western countries (WendelVos et al. 2007). Studies on physical exercise behaviors indicate that cardiovascular disease, hypertension, high cholesterol, low bone density, obesity and low physical fitness are linked with sedentary lifestyle (Davison \& Lawson, 2006; Sheu-jen, Wen-chi, Patricia, \& Jackson, 2010). It is also reported that physical inactivity is an important cause of diabetes and certain types of cancers (United States Department of Health and Human Services, 1996). Moreover, people with inactive habit of exercise are deprived of positive social and emotional exchanges that generally help to reduce anxiety, stress and other psychological problems (Hassmén, Koivula, \& Uutela, 2000).

Only a small portion of people are able to meet up the recommended physical activities. Around 14\%
American young people are completely inactive and another $35 \%$ of youth fail to meet the minimum activity guidelines (Davison \& Lawson, 2006). Only 29\% of women, aged 45 to 64 , meet the Surgeon General's recommendations of 30 minutes of activities per week (King et al. 2005). The negative health impacts from physical inactivity have aroused international efforts to prioritize activity promotion (Sallis et al. 2009).

Built environment may provide stimulus or barriers to people's participation in physical activities. Prior studies indicate that positive environmental intervention forms a strategic approach in promoting physical activities (Leslie et al. 2007). Links between elements of physical environment and active lifestyle have been confirmed by a wide range of studies. In order to encourage physical activity, insights into factors that facilitate or inhibit physical activity need to be examined. Despite there are a couple of reviews on potential environmental attributes on physical activities, a systematical review on attributes of built environment 
shaping physical activities and approaches to measure those attributes are less documented. In addition, this article argues that integrated measures of built environment might be helpful to reduce limitations of individual measures and to understand the reasons of less participation in physical activities. However, specifically, this paper summarizes the environmental factors that affect physical activities and the state of the arts of the ways used to measure those factors. Moreover, it tries to identify limitations of current studies related to built environment, and shed lights on future research directions. It hopes to provide suggestions for environmental interventions in landscape and urban planning to promote physical activity behaviors.

This paper is divided into four sections. The second section provides a systematic summary of different characteristics of built environment that are associated with physical activities. The third section reviews the methods used to measure those characteristics. The fourth section points out several future research directions. And some environmental interventions are proposed in the concluding section.

\section{Characterizing built environment}

There are researches regarding environmental attributes of built environment in relation to physical activities. Based on a comprehensive literature review, this section tries to summarize characteristics of built environment. The characteristics can be categorized into six sub-headings viz. non-motorized transport infrastructure, destination distribution, safety, landform, local amenities and others (Figure 1).

\subsection{Non-motorized transport infrastructure}

There are researches in transportation studies investigate human health issues, mainly focusing on traffic-related injuries and automobile related air pollution (Sallis, Frank, Saelens, \& Kraft, 2004). An increasing number of them have also integrated methods in behavioral research into the health and urban planning researches to examine the connection between physical activities and non-motorized transportation infrastructures (King et al. 2002). It is also reported that physical activity is associated with presence of sidewalks, bicycle trails and the inclusive of walking route (Craig et al. 2002). The presence of bike-lanes is also correlated with physical activity (Inoue et al. 2009). Improvement in cycling infrastructure, such as off-road bicycle lanes is likely to increase cycling among underrepresented population such as women (Garrard, Rose, \& Lo, 2008). Quality and maintenance of the sidewalk is also examined among the elderly population (Gómez et al. 2010). Some of them also suggest that adequate sidewalks and people-oriented public walking tracks especially for all ages and both genders are important factors that may encourage physical activity (Gallagher et al. 2010).

\section{Destination distributions}

Six domains of destinations are summarized based on a research by Witten et al. (2011) (Figure 1). Recreational destinations may be considered as the convergences of physical activities around neighborhoods, for instance basketball courts, golf courses, parks, martial arts studios, playing fields, tracks, skating rinks, swimming pools, tennis courts and gymnastic clubs (Baker et al. 2008; Scott et al. 2007). Educational destinations include schools, universities and daycare centers (Witten et al. 2011). Transport destinations comprise ports, bus stops and railway stations (Brown \& Werner 2009; Rietveld 2000). Social and cultural destinations are the places where people can meet and share interest, including mosques, temples, churches, cafes and bars. Pfeiffer et al. (2011) investigate the association of physical activity and church availability, church attendance, and find a positive correlation between physical activity and the proximity to and use of churches. Shopping and retail facilities are the places where daily needs can be met, such as food retails and shopping centers. Zick et al. (2009) state that the availability of food stores in the neighborhood is correlated with physical activities, which may reduce the risk of obesity. The last domain is service destinations, where services are provided to facilitate daily life, such as hospital, bank, and post office etc. Distance to major services is likely to influence walking trip in local neighborhood (Leslie et al. 2007).

However, destination distributions have been accounted for three aspects: accessibility, intensity and patterns (Brownson et al. 2009). Accessibility refers to road network which enables individual to reach destinations (Geurs \& van Wee 2004). Access to a variety of destinations has been examined by previous studies (Coombes et al. 2010). It was found that the size and attractiveness of facilities were also incorporated as characteristics of each destination when evaluating accessibility (Lackey \& Kaczynski 2009). Intensity stands for the number or proportion of destinations in a certain area. Recreational resources are examined by counting the number of destinations in certain geographic area (Baker et al. 2008). And finally, patterns indicate the degree the evenness or diversity of different destinations in a certain area, which can be quantified by balance index or entropy measures (Brownson et al. 2009). 


\section{Safety}

Safe environment is a critical indicator for physical activity, which has got much attention of prior studies. Safety with regard to physical activity was mainly investigated in two aspects, viz. traffic hazard and crime threat. Traffic-safety related infrastructures, such as pedestrian lights, speed humps, speed limit and traffic volume have been proved to be very important in promoting physical activity (Carver et al. 2010). Foster \& Giles-Corti (2008) also find fear of crime including vandalism, robbery, burglary and rape in the neighborhood which are correlated with tendency of less outdoor activities. In addition, there are studies examine categories of susceptibility to different slice of populations, such as adolescent, old adults, pregnant women (Foster \& Giles-Corti 2008; Mudd, et al. 2009; Tucker-Seeley et al. 2009).

\section{Landform}

Characteristics of physical landform have direct and indirect relations with human health. Several dimensions of landform have been examined in previous researches (Sallis et al. 2004; Leslie et al. 2007; Gomez et al. 2010; Xu et al. 2010). The dimensions include road connectivity, land use mix, slope and residential density (Figure 1). Connectivity represents the degree that destinations are linked together based on road network. Highly connected road network provides greater potential routes and convenience to reach destination (Leslie et al. 2007). Land use mix measures the degree of hybrid living space, which signifies the extent of reliance on automobile. It was found that land use mix is related to greater walking or cycling among residents (Sallis et al. 2004). Slope of the land is found to be negatively correlated with walking of elderly people. Gómez et al. (2010) note that people who live in areas with slope greater than 5\% are less likely to walk more than 60 minutes. Residential density is another important indicator of neighborhood built environment. Higher density of residents normally implies more destinations and increased social capitals, which facilitates walking and cycling within the communities. Nonetheless, both negative and positive relationship between physical activity and residential density are reported in previous studies (Kligerman et al. 2007; Xu et al. 2010).

\section{Local amenities}

There are local amenities that have also been investigated to understand their significance in enhancing physical activity (Figure 1). Visual aesthetics of neighborhood such as color, texture, composition, configuration, attractiveness, visual interests and variety and enjoyable scenery are associated with walking (Craig et al. 2002; Inoue et al. 2009; Mota et al. 2005). Greenness is another local amenity, which characterizes neighborhood environment and provides opportunities of outdoor physical activity for the residents (Leslie, Sugiyama, Ierodiaconou, \& Kremer, 2010). Coastal environment may also be an important stimulus of physical activity. It was found that the residents stay in the coastal locations walk considerably more minutes in their neighborhoods than others (Humpel et al. 2004).

\section{Others}

Besides the factors discussed above, there are several neighborhood characteristics, which are found to be correlated with physical activity. These are population density, urban sprawl, weather conditions, and blighted areas. Sallis et al. (2004) show that density of population has a consistant positive correlation with walking behaviour. It was also explored that urban sprawl fairly correlates with walking minutes, implying that urbanization may increase non-motorized mobility (Ewing et al., 2003). There are evidences also indicate that weather condition is associated with physical activity (Davison \& Lawson 2006; Humpel et al. 2004). In addition, blighted areas also decrease the tendency of walking and cycling in the neighborhood. For example, presence of unattended dogs, air and noise pollution and home-age are correlated with neighborhood walking or cycling (Berrigan \& Troiano 2002; WendelVos et al. 2007).

\section{Measuring built environment}

For an authentic characterization of different aspects of built environment, developing high quality and suitable measures is pivotal (Sallis et al. 2000). Brownson et al. (2009) state three categories of measures for characterizing physical environment: (i) perceived environment measures, (ii) observational measures and (iii) GIS-based measures. Drawing upon these characterizations, this section reviews existing measures of environmental attributes in the light of characteristics of built environment discussed in section two. 


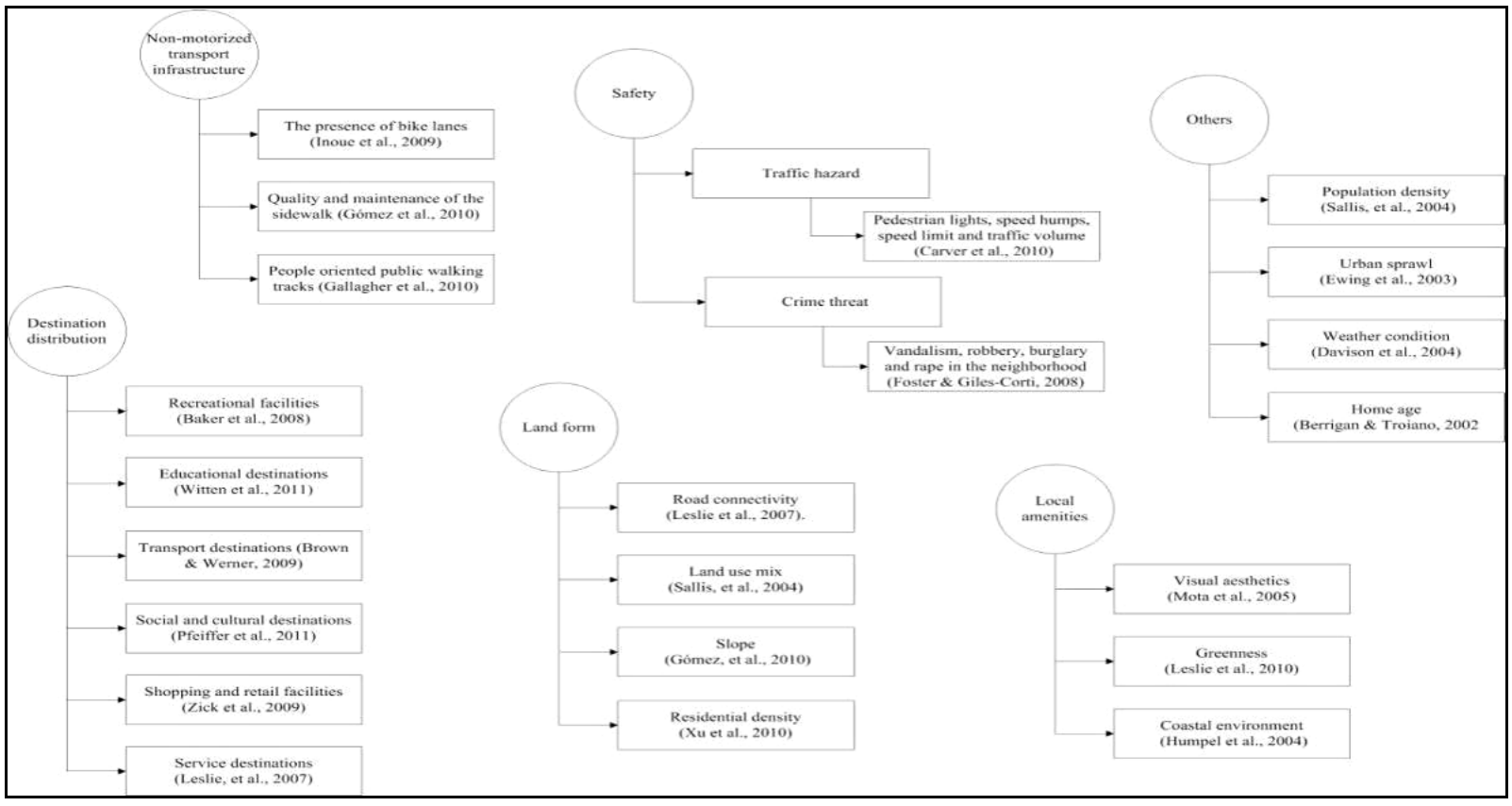

Figure 1: Attributes of built environment and physical activities

\section{Perceived environment measures}

Questionnaires and interviews are two common ways to collect respondents' perceptions about the neighborhood environment. Interviews are normally conducted in the form of in-person or telephone interview while questionnaires are mostly distributed in certain locations or dispatched by mails. Both ways have been used to demonstrate associations between physical-environmental characteristics, such as accessibility with recreational facilities and aesthetics (Mota et al. 2005), and neighborhood safety with physical activity (Mudd et al. 2009). A couple of perceived physical environment including land use mix condition, available facilities, traffic and crime safety, aesthetics, street connectivity and residential density were surveyed in different cities, based on a composite index which was calculated to represent the general neighborhood environment (Rosenberg et al. 2009; Sheu-jen et al. 2010).

Both questionnaires and interviews are important tools for reflecting residents' perception about their living environment and for unveiling their everyday experience, adaptation and interaction with natural spaces. Nonetheless, self-reported neighborhood characteristics based on interview methods may vary largely because of individual economic and sociocultural differences. In addition, the non-response rate may correlate with the length of the questionnaires and number of respondents attended in the survey (Brownson et al. 2009). In addition, it is very difficult to measure spatial attributes of perceived environment. This might be a reason why many researches ignore spatial dimension of neighborhood environment.

\section{Observational measures}

Environmental audit of the qualities of environment in relation to physical activities is another important and frequent-used observational measure (Brownson et al. 2009). Systematic on-site observation can be used to record different aspects of physical environmental characteristics, such as accessibility and pleasurability (Franzini et al. 2010); and traffic, sidewalk, and aesthetics (Suminski et al. 2008). Since the in-person observation of physical environment is labor-intensive, and time-consuming to conduct; observation requires some knowledge and practical experiences in the relevant fields. Several recent studies tried to "remotely" audit physical environment using some technical platforms, such as Google Earth and Google Map Street View (Rundle et al. 2011; Taylor et al. 2011), which dramatically increase the efficiency of measuring physical environment. Thus it seems that this type of observational measure might be a significant method of collecting and analyzing environment-related data. Nevertheless, it should also be remembered that the observational analysis with an extensive item of phenomena may be very specialized and complex technical process. 


\section{GIS-based measures}

Geographic information system has been widely used to characterize physical environment because of its advantage in processing data in different geographic scale, performing powerful spatial analysis and facilitating result visualization and interpretation. Characteristics of neighborhood destination, landform and transportation are the common variables measured using GIS (Coombes et al. 2010; Witten et al. 2011). Safety was also mapped out using participatory GIS method (Wridt 2010). Composite score, based on the generalization of each individual attribute such as density, connectivity, land use and retail area, was also calculated in GIS environment (Leslie et al. 2007).

GIS can be used as a good environment surveillance tool as it has been adopted in many objective measures. To implement GIS, spatial data are required to support spatial analysis. However, data incompleteness and inaccuracy may perplex the process of spatial auditing. Determination of analysis scale and boundary of neighborhood entails cautions because arbitrarily determined neighborhood boundary may not be representative to capture the relationship between the neighborhood environment and physical activity.

\section{Integration of different measures}

The above mentioned discussion on three measures notes strengths and weakness of their practical applications. There are studies critically scrutinize individual use of these measures. Several studies argue that integration of these measures is important to reduce their individual flaws and increase their usability. This section briefly reviews few literatures to show integrative uses of different measures.

Researches, in the area of both public health and urban planning, highlight the role of objective measure of physical environment in understanding physical activity behaviors (Leslie et al. 2007). Despite the presence of physical activity promoting built environment/ infrastructure is important in shaping people's lifestyle. On the contrary previous studies suggest that only infrastructural development may not be sufficient and effective to encourage physical activity without sociocultural awareness (Gebel et al., 2011). Therefore, both the presence of built environment and a positive perception of the residents are important variables in studying neighborhood environment. Realizing this fact, several studies employed both perceived environmental measures and observational or GIS-based measures in their research design (Boehmer et al. 2006; Franzini et al. 2010; Tilt et al. 2007). Nevertheless, there are studies pay less attention to combining perceived and objective measures of neighborhood attributes and physical activity behavior (Boehmer et al. 2006). The following section describes existing practical and potential environmental interventions in promoting physical activity.

\section{Improving built environment: practical interventions and physical activity}

The review finds different infrastructural interventions and factors in stimulating engagement in physical activities. These include improving non-motorized transport infrastructure, increasing destination accessibility, reenforcing neighborhood safety, optimizing landform, increasing local amenities, and shape active perception. Figure 2 offers an integrated framework, which shows interaction between characteristics of built environment and interventions for improving engagement in physical activities. This section particularly focuses on the different infrastructural interventions, which are as following:

\section{Improving non-motorized transport infrastructure}

Environmental interventions constitute one of the crucial approaches in promoting participation in physical activity (Leslie et al. 2007). Design appropriate form of activity-friendly interventions that cater to most neighborhood residents are very important in urban planning and landscape design. There are evidences that environment-friendly non-motorized transport infrastructure is certainly important to promote non-motorized travel behavior (McMillan 2007). Strategies such as separating pedestrians and cycling trails from vehicle lanes and linking those nonmotorized tracks with public transport might be helpful in encouraging physical activity.

\section{Increasing accessibility}

Walking access to everyday destinations is an important factor in determining population level of physical activity (Witten et al. 2011). For example, developing full-service grocery stores within walking distance in residential areas and incorporate farmers' market as a complement into local community are suggested to facilitate neighborhood walking (Department of Design and Construction, 2010).

\section{Reinforcing neighborhood safety}

Traffic hazard and crime problem affect residents' participation in outdoor activity (Carver et al. 2010; Foster \& Giles-Corti 2008). Interventions such as trafficcalming practices, such as lowering speed limit and raising road hump in residential areas will upgrade traffic safety level, thereafter encourage outdoor activity. These interventions are particularly important for children and elderly people. Reinforcing surveillance of neighborhoods and maintenance of facilities might effectively reduce crime ensuring a safe active space. 


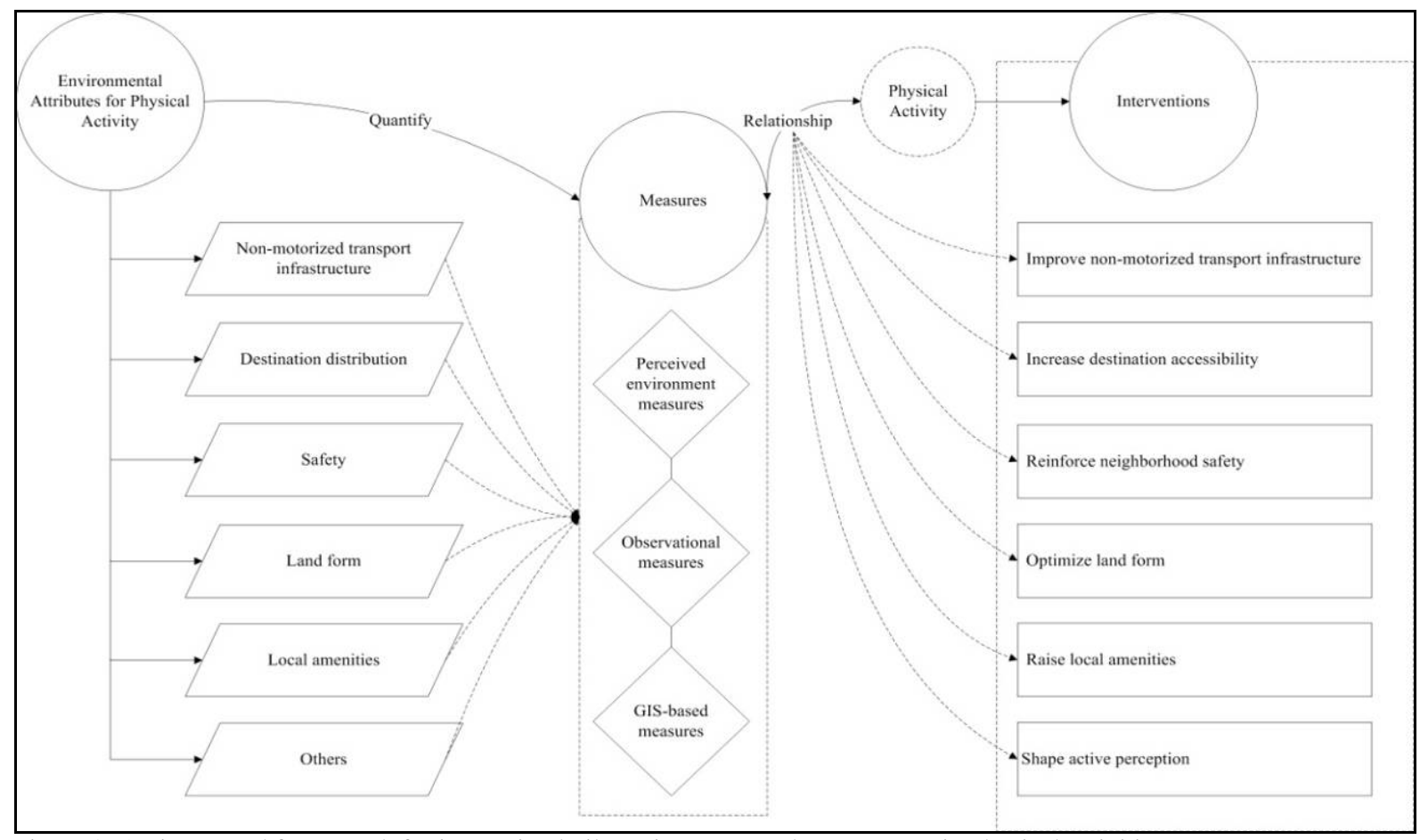

Figure 2: An integrated framework for improving built environment and engagement in physical activities

\section{Optimizing landform}

Attributes of landform such as density, land use mix, connectivity are robustly linked with physical activity (Leslie et al. 2007). Planners should emphasize on a well connected road network and hybrid land use when developing new neighborhood. For existing neighborhoods in which street connection is poor; construction of pedestrian paths linking residential blocks might be a good strategy to enhance people participation in walking, cycling and other physical activities (Department of Design and Construction, 2010).

\section{Increasing local amenities}

Attractive neighborhood environment is a stimulus of outdoor activity (Craig et al. 2002). Creating intriguing and aesthetic spots in the neighborhoods can effectively inspire people for exercise and interaction. Open spaces with activity facilitators such as benches and lights provide place to rest (Xiaolu and Rana 2012). Cafes or churches in close proximity encourage people to interact and communicate. These places are important local amenities to accrue social capital, eventually forming a healthy neighborhood.

\section{Shape active perception}

Prior studies suggest that unawareness or underestimate of positive neighborhood environment limits physical activity behaviors (Gebel et al. 2011). Besides creating more positive feature, making residents to realize neighborhood amenities and inspiring a spontaneously active lifestyle is crucial to promote physical activity. Social events such as monthly cycling, community hiking and collective outdoor exercises can be organized to raise awareness of neighborhood as well as importance of physical activity.

\section{Conclusion}

The article aims to demonstrate relations between physical activities and built environment. It was argued that built environment may act as stimulus or barriers of physical activities. Based on this argument, this article suggests that understanding physical attributes of built environment is important to enhance participation in physical activities. In addition, there are studies emphasize only a single measure of built environment, which undoubtedly miss out some other important variables. For this reason, it is hoped that the integrated measures of build environment and their relations with infrastructural interventions, articulated in this article, might be helpful in improving neighborhood environment as a participatory social and secured space. Above all, the integrated framework, which includes characteristics of build environment with respect to physical activities, measures of built environment, and options of practical interventions, may also be conducive in spatial planning and development of built environment. Moreover, the framework recommends some practical interventions for improvement of build environment which is in fact inevitable to encourage physical activities. 


\section{References}

Baker, E. A., Schootman, M., Kelly, C., \& Barnidge, E. (2008). Do recreational resources contribute to physical activity? Journal of Physical Activity and Health, 5(2), 252-261.

Berrigan, D., \& Troiano, R. P. (2002). The association between urban form and physical activity in U.S. adults. American Journal of Preventive Medicine, 23(2 SUPPL. 1), 74-79.

Boehmer, T. K., Hoehner, C. M., Wyrwich, K. W., Brennan Ramirez, L. K., \& Brownson, R. C. (2006). Correspondence Between Perceived and Observed Measures of Neighborhood Environmental Supports for Physical Activity. Journal of Physical Activity \& Health, 3(1), 22-36.

Boone-Heinonen, J., Popkin, B. M., Song, Y., \& Gordon-Larsen, P. (2010). What neighborhood area captures built environment features related to adolescent physical activity? Health and Place, 16(6), 1280-1286.

Brennan-Horley, C., \& Gibson, C. (2009). Where is creativity in the city? Integrating qualitative and GIS methods. Environment and Planning A, 41(11), 2595-2614.

Brennan-Horley, C., Luckman, S., Gibson, C., \& Willoughby-Smith, J. (2010). Gis, ethnography, and cultural research: Putting maps back into ethnographic mapping. Information Society, 26(2), 92-103.

Brown, B., \& Werner, C. (2009). Before and after a new light rail stop. Journal of the American Planning Association, 75(1), 5-12.

Brownson, R. C., Hoehner, C. M., Day, K., Forsyth, A., \& Sallis, J. F. (2009). Measuring the Built Environment for Physical Activity. State of the Science. American Journal of Preventive Medicine, 36(4 SUPPL.), S99-S123.e112.

Carver, A., Timperio, A., Hesketh, K., \& Crawford, D. (2010). Are safety-related features of the road environment associated with smaller declines in physical activity among youth? Journal of Urban Health, 87 (1), 29-43.

Coombes, E., Jones, A. P., \& Hillsdon, M. (2010). The relationship of physical activity and overweight to objectively measured green space accessibility and use. Social Science and Medicine, 70(6), 816-822.

Craig, C. L., Brownson, R. C., Cragg, S. E., \& Dunn, A. L. (2002). Exploring the effect of the environment on physical activity: A study examining walking to work. American Journal of Preventive Medicine, 23 (2 SUPPL. 1), 36-43.

Davison, K. K., \& Lawson, C. T. (2006). Do attributes in the physical environment influence children's physical activity? A review of the literature. International Journal of Behavioral Nutrition and Physical Activity, 3.

Department of Design and Construction. (2010). Active design guidelines promoting physical activity and health in design. Retrived from http://www.nyc.gov/html/ddc/html/design/active_design.shtml

Ewing, R., Schmid, T., Killingsworth, R., Zlot, A., \& Raudenbush, S. (2003). Relationship between urban sprawl and physical activity, obesity, and morbidity. American Journal of Health Promotion, 18(1), 47-57.

Forsyth, A., Hearst, M., Oakes, J. M., \& Schmitz, K. H. (2008). Design and destinations: Factors influencing walking and total physical activity. Urban Studies, 45 (9), 1973-1996.

Foster, S., \& Giles-Corti, B. (2008). The built environment, neighborhood crime and constrained physical activity: An exploration of inconsistent findings. Preventive Medicine, 47(3), 241-251.

Franzini, L., Taylor, W., Elliott, M. N., Cuccaro, P., Tortolero, S. R., Janice Gilliland, M., Grunbaum J, Schuster MA. (2010). Neighborhood characteristics favorable to outdoor physical activity: Disparities by socioeconomic and racial/ethnic composition. Health and Place, 16(2), 267-274.
Gallagher, N. A., Gretebeck, K. A., Robinson, J. C., Torres, E. R., Murphy, S. L., \& Martyn, K. K. (2010). Neighborhood factors relevant for walking in older, Urban, African American adults. Journal of Aging and Physical Activity, 18(1), 99-115.

Garrard, J., Rose, G., \& Lo, S. K. (2008). Promoting transportation cycling for women: The role of bicycle infrastructure. Preventive Medicine, 46(1), 55-59.

Gebel, K., Bauman, A. E., Sugiyama, T., \& Owen, N. (2011). Mismatch between perceived and objectively assessed neighborhood walkability attributes: Prospective relationships with walking and weight gain. Health and Place, 17(2), 519-524.

Geurs, K. T., \& van Wee, B. (2004). Accessibility evaluation of landuse and transport strategies: Review and research directions. Journal of Transport Geography, 12(2), 127-140.

Gómez, L. F., Parra, D. C., Buchner, D., Brownson, R. C., Sarmiento, O. L., Pinzon, J. D., Ardila, M., Moreno, J., Serrato, M., Lobelo, F. (2010). Built Environment Attributes and Walking Patterns Among the Elderly Population in Bogotá. American Journal of Preventive Medicine, 38(6), 592-599.

Hassmén, P., Koivula, N., \& Uutela, A. (2000). Physical exercise and psychological well-being: A population study in Finland. Preventive Medicine, 30(1), 17-25.

Hillsdon, M., Panter, J., Foster, C., \& Jones, A. (2006). The relationship between access and quality of urban green space with population physical activity. Public Health, 120(12), 1127-1132.

Humpel, N., Owen, N., Iverson, D., Leslie, E., \& Bauman, A. (2004). Perceived environment attributes, residential location, and walking for particular purposes. American Journal of Preventive Medicine, 26(2), 119-125.

Huynh, N. T., \& Doherty, S. T. (2007). Digital sketch-map drawing as an instrument to collect data about spatial cognition. Cartographica, 42(4), 285-296.

Inoue, S., Murase, N., Shimomitsu, T., Ohya, Y., Odagiri, Y., Takamiya, T., Kaori I., Toshihito K., James F. S. (2009). Association of physical activity and neighborhood environment among Japanese adults. Preventive Medicine, 48(4), 321-325.

King, A. C., Stokols, D., Talen, E., Brassington, G. S., \& Killingsworth, R. (2002). Theoretical approaches to the promotion of physical activity: Forging a transdisciplinary paradigm. American Journal of Preventive Medicine, $23(2$ SUPPL. 1), 15-25.

King, W. C., Belle, S. H., Brach, J. S., Simkin-Silverman, L. R., Soska, T., \& Kriska, A. M. (2005). Objective measures of neighborhood environment and physical activity in older women. American Journal of Preventive Medicine, 28(5), 461-469.

Kligerman, M., Sallis, J. F., Ryan, S., Frank, L. D., \& Nader, P. R. (2007). Association of neighborhood design and recreation environment variables with physical activity and body mass index in adolescents. American Journal of Health Promotion, 21(4), 274-277.

Kwan, M. P. (1999). Gender and individual access to urban opportunities: A study using space-time measures. Professional Geographer, 51(2), 210-227.

Lackey, J. L., \& kaczynski, A. T. (2009). Correspondence of perceived vs. objective proximity to parks and their relationship to park-based physical activity. International Journal of Behavioral Nutrition and Physical Activity, 6.

Leitão, A. B., Miller, J., Ahern, J., \& McGarigal, K. (2006). Measuring landscapes : a planner's handbook. Washington, DC: Island Press.

Leslie, E., Coffee, N., Frank, L., Owen, N., Bauman, A., \& Hugo, G. (2007). Walkability of local communities: Using geographic information systems to objectively assess relevant environmental attributes. Health and Place, 13(1), 111-122.

Leslie, E., Sugiyama, T., Ierodiaconou, D., \& Kremer, P. (2010). Perceived and objectively measured greenness of neighbourhoods: Are they measuring the same thing? Landscape and Urban Planning, 95(1-2), 28-33. 
Maddison, R., Jiang, Y., Hoorn, S. V., Mhurchu, C. N., Exeter, D., \& Utter, J. (2010). Perceived versus actual distance to local physical-activity facilities: Does it really matter? Journal of Physical Activity and Health, 7(3), 323-332.

Matei, S., Ball-Rokeach, S. J., \& Qiu, J. L. (2001). Fear and misperception of Los Angeles urban space: A spatial-statistical study of communication-shaped mental maps. Communication Research, 28(4), 429-463.

McMillan, T. E. (2007). The relative influence of urban form on a child's travel mode to school. Transportation Research Part A: Policy and Practice, 41(1), 69-79.

Mondschein, A., Blumenberg, E., \& Taylor, B. (2010). Accessibility and cognition: The effect of transport mode on spatial knowledge. Urban Studies, 47(4), 845-866.

Mota, J., Almeida, M., Santos, P., \& Ribeiro, J. C. (2005). Perceived Neighborhood Environments and physical activity in adolescents. Preventive Medicine, 41(5-6), 834-836.

Mudd, L. M., Nechuta, S., Pivarnik, J. M., \& Paneth, N. (2009). Factors associated with women's perceptions of physical activity safety during pregnancy. Preventive Medicine, 49(2-3), 194-199.

Pfeiffer, K., Colabianchi, N., Dowda, M., Porter, D., Hibbert, J., \& Pate, R. R. (2011). Examining the role of churches in adolescent girls' physical activity. Journal of Physical Activity and Health, 8(3), 227-233.

Rietveld, P. (2000). The accessibility of railway stations: The role of the bicycle in The Netherlands. Transportation Research Part D: Transport and Environment, 5(1), 71-75.

Rosenberg, D., Ding, D., Sallis, J. F., Kerr, J., Norman, G. J., Durant, N., Harris, S. K., Saelens, B. E. (2009). Neighborhood Environment Walkability Scale for Youth (NEWS-Y): Reliability and relationship with physical activity. Preventive Medicine, 49(2-3), 213-218.

Rundle, A. G., Bader, M. D. M., Richards, C. A., Neckerman, K. M., \& Teitler, J. O. (2011). Using google street view to audit neighborhood environments. American Journal of Preventive Medicine, 40(1), 94-100.

Sallis, J. F., Bowles, H. R., Bauman, A., Ainsworth, B. E., Bull, F. C., Craig, C. L., Michael S., Ilse De B., Johan L., Victor M., Sandra M., Duncan J. M., Luis F. G., Shigeru I., Norio M., Vida V., Grant M., Harriette C., Lena K.H., Heidi T., Patrick B. (2009). Neighborhood Environments and Physical Activity Among Adults in 11 Countries. American Journal of Preventive Medicine, 36(6), 484-490.

Sallis, J. F., Frank, L. D., Saelens, B. E., \& Kraft, M. K. (2004). Active transportation and physical activity: Opportunities for collaboration on transportation and public health research. Transportation Research Part A: Policy and Practice, 38(4), 249-268.

Sallis, J. F., Owen, N., \& Fotheringham, M. J. (2000). Behavioral epidemiology: A systematic framework to classify phases of research on health promotion and disease prevention. Annals of Behavioral Medicine, 22(4), 294-298.

Scott, M. M., Evenson, K. R., Cohen, D. A., \& Cox, C. E. (2007). Comparing perceived and objectively measured access to recreational facilities as predictors of physical activity in adolescent girls. Journal of Urban Health, 84(3), 346-359.
Sheu-jen, H., Wen-chi, H., Patricia, A. S., \& Jackson, P. W. (2010). Neighborhood environment and physical activity among Urban and Rural Schoolchildren in Taiwan. Health and Place, 16(3), 470-476.

Suminski, R. R., Heinrich, K. M., Poston, W. S. C., Hyder, M., \& Pyle, S. (2008). Characteristics of urban sidewalks/streets and objectively measured physical activity. Journal of Urban Health, 85(2), 178-190.

Taylor, B. T., Fernando, P., Bauman, A. E., Williamson, A., Craig, J. C., \& Redman, S. (2011). Measuring the quality of public open space using google earth. American Journal of Preventive Medicine, 40(2), 105-112.

Tilt, J. H., Unfried, T. M., \& Roca, B. (2007). Using objective and subjective measures of neighborhood greenness and accessible destinations for understanding walking trips and BMI in Seattle, Washington. American Journal of Health Promotion, 21(4 SUPPL.), 371-379.

Tucker-Seeley, R. D., Subramanian, S. V., Li, Y., \& Sorensen, G. (2009). Neighborhood Safety, Socioeconomic Status, and Physical Activity in Older Adults. American Journal of Preventive Medicine, 37(3), 207-213.

Turner, M. G., Gardner, R. H., \& O'Neill, R. V. (2001). Landscape ecology in theory and practice: Pattern and Process. New York: Springer.

Weber, J. (2003). Individual accessibility and distance from major employment centers: An examination using space-time measures. Journal of Geographical Systems, 5(1), 51-70.

Wendel-Vos, W., Droomers, M., Kremers, S., Brug, J., \& Van Lenthe, F. (2007). Potential environmental determinants of physical activity in adults: A systematic review. Obesity Reviews, 8(5), 425-440.

Witten, K., Pearce, J., \& Day, P. (2011). Neighbourhood destination accessibility index: A GIS toolfor measuring infrastructure support for neighbourhood physical activity. Environment and Planning A, 43(1), 205-223.

Wridt, P. (2010). A qualitative gis approach to mapping urban neighborhoods with children to promote physical activity and child-friendly community planning. Environment and Planning B: Planning and Design, 37(1), 129-147.

Xiaolu, Zhou and Rana, Md. Masud Parves (2012). Social benefits of urban green space: a conceptual framwork of valuation and accessibility measurements. Managemnet of Environmental Quality: An International Journal. Vol. 23, No.. 2, 173-189.

Xu, F., Li, J., Liang, Y., Wang, Z., Hong, X., Ware, R. S., Eva L., Takemi S., and Neville O. (2010). Associations of residential density with adolescents' physical activity in a rapidly urbanizing area of mainland China. Journal of Urban Health, 87(1), 44-53.

Zick, C. D., Smith, K. R., Fan, J. X., Brown, B. B., Yamada, I., \& Kowaleski-Jones, L. (2009). Running to the Store? The relationship between neighborhood environments and the risk of obesity. Social Science and Medicine, 69(10), 1493-1500.

Manuscript received on 27 September 2012 and revised on 28 December 2012 